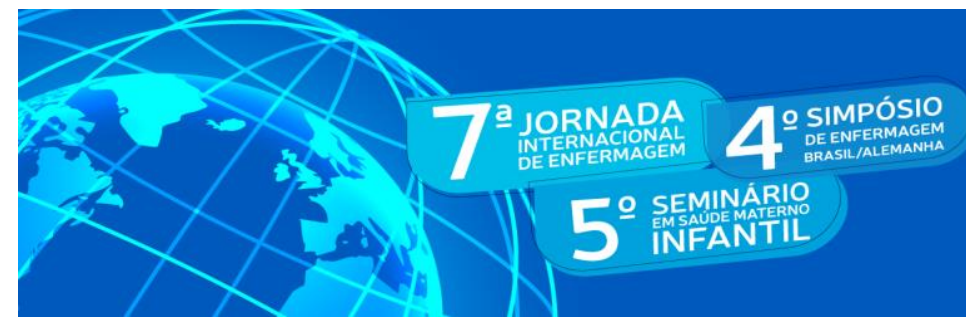

PRÁTICA AVANÇADA EM SAŨDE:

desofios e projeçōes interprofissionais

05 a 07.05 de 2021

ISSN: 2318-0234

DOI: http://doi.org/10.48195/jie2021-145

\title{
QUALIFICAÇÃO EM BOAS PRÁTICAS PARA MANIPULADORES DE ALIMENTOS: UM RELATO DE EXPERICÊNCIA. ${ }^{1}$
}

\section{Amanda Vargas Spat ${ }^{2}$; Elaine Ferreira de Araújo³; Isabela Rodrigues Barroso ${ }^{4}$; Patrícia Arruda Scheffer ${ }^{5}$; Ana Lúcia de Freitas Saccol ${ }^{6}$}

\begin{abstract}
RESUMO
O presente estudo teve como objetivo relatar a experiência de acadêmicos do curso de nutrição da Universidade Franciscana de Santa Maria, que contou com a participação do Mestrado em Ciências da Saúde e da Vida, na disciplina extensionista de Qualidade e Segurança dos Alimentos. A atividade desenvolvida trata-se de uma Qualificação Online em Boas Práticas para manipuladores de alimentos e pequenos produtores de Santa Maria, utilizando como embasamento científico a legislação federal e estadual vigente. $\mathrm{O}$ evento realizado em janeiro de 2021 contou com atestado de participação e pesquisa de satisfação utilizando a escala Likert. O estudo mostrou que, apesar de imprevistos de conexão de alguns alunos, a capacitação ocorreu sem prejudicar a qualidade dos temas abordados, pois todos os alunos envolvidos estavam a par das temáticas de BPM. Além disso, a pesquisa de satisfação confirmou a expectativa dos organizadores, pois demonstrou excelente aceitabilidade pelos inscritos.
\end{abstract}

Palavras-chave: Relato de Experiência; Nutrição; Extensão; Capacitação.

\begin{abstract}
This study aimed to report the experience of academics in the nutrition course at Universidade Franciscana of Santa Maria, which included the participation of the Master in Health and Life Sciences, in the extension discipline of Quality and Food Safety. The activity developed is an Online Qualification in Good Practices for food handlers and small producers in Santa Maria, using the current federal and state legislation as a scientific basis. The event held in January 2021 featured a certificate of participation and a satisfaction survey using the Likert scale. The study showed that, despite the unforeseen connection issues suffered by some students, the training took place without impairing the quality of the topics covered, as all students involved were familiar with the topics of Good Practices in Food Handling. Furthermore, the satisfaction survey confirmed the expectations of the organizers, as it demonstrated excellent acceptability by the enrolled.
\end{abstract}

Key Words: Experience Reporting; Nutrition; Extension; Training.

\footnotetext{
${ }^{1}$ Trabalho desenvolvido na disciplina extensionista de Qualidade e Segurança dos Alimentos no curso de Nutrição da Universidade Franciscana

${ }^{2}$ Estudante do Curso de Nutrição. Universidade Franciscana. E-mail: amanda.spat@ufn.edu.br

${ }^{3}$ Estudante do Curso de Nutrição. Universidade Franciscana. E-mail: elaine.ferreira@ufn.edu.br

${ }^{4}$ Estudante do Curso de Nutrição. Universidade Franciscana. E-mail: isabela.barroso@ufn.edu.br

${ }^{5}$ Nutricionista. Mestranda em Ciências da Saúde e da Vida- Universidade Franciscana. Grupo de Pesquisa em Segurança Alimentar e Nutricional (GESAN/CNPq) E-mail: patricia.scheffer@ufn.edu.br

6 Orientadora. Docente no curso de Nutrição e Mestrado em Ciências da Saúde e da Vida- Universidade Franciscana. Grupo de Pesquisa em Segurança Alimentar e Nutricional (GESAN/CNPq) E-mail: alsaccol@prof.ufn.edu.br
} 


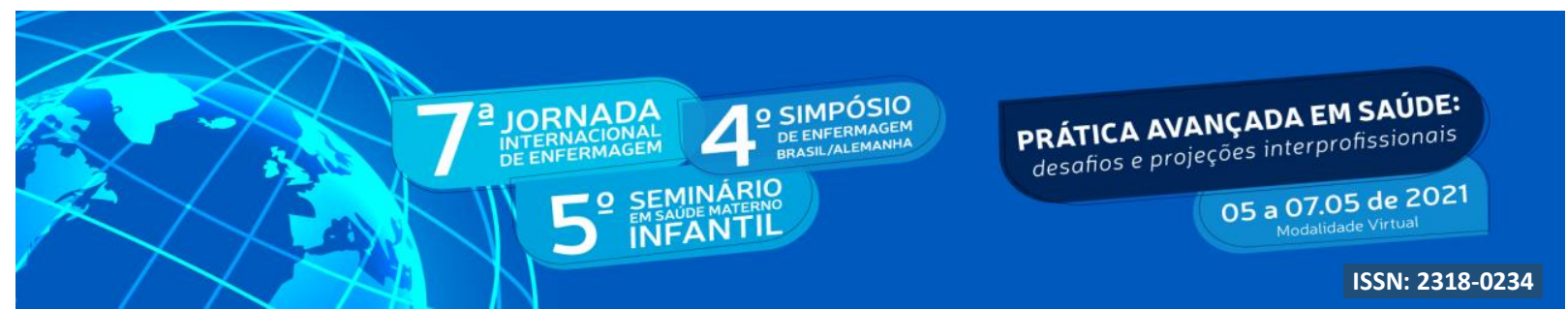

\section{INTRODUÇÃ̃O}

Compreende-se por Serviço de alimentação os locais ou empresas que fornecem, preparam ou comercializam alimentos e preparações feitas fora do lar. Segundo a Resolução da Diretoria Colegiada (RDC) n 216 de 2004, de âmbito nacional, define os serviços de alimentação como: "estabelecimentos onde o alimento é manipulado, preparado, armazenado e ou exposto à venda, podendo ou não ser consumido no local” (BRASIL, 2004).

Em virtude da globalização, a crescente busca por alimentação fora do lar, tornou-se cada vez mais frequente (BEZZERA et al, 2016). No Brasil, de acordo com últimos dados disponíveis pela Associação Brasileira das Empresas de Refeições Coletivas (ABERC) o consumo de refeições de 2012 para 2019 aumentou cerca de 4 milhões (ASSOCIAÇÃO BRASILEIRA DE EMPRESAS DE REFEIÇÕES COLETIVAS, 2020). Sendo assim o controle da qualidade e segurança do alimento nos serviços de alimentação são de extrema importância para a saúde da população, tendo vista que o alimento inadequadamente manipulado pode causar inúmeros problemas na saúde do consumidor.

Dentre eles, destaca-se as Doenças Transmitidas por Alimentos (DTA), que representam mais de 250 tipos em todo o mundo. As DTA podem ser causadas pela ingestão de água ou de alimentos contaminados, gerando infecções por toxinas, bactérias, vírus ou parasitas. Segundo informações do Sistema de Informação e Agravos de Notificação (Sinan), há uma média anual de 700 surtos de DTA, cerca de 13 mil doentes (BRASIL,2020).

Segundo Taniwaki et al. (2020), é imprescindível que os manipuladores de alimentos obtenham capacitação técnica em Boas Práticas de Manipulação (BPM) para aprimorar suas habilidades e garantir a qualidade e segurança higiênico-sanitária das preparações e do ambiente de trabalho. Para garantir esta qualidade e segurança, a Agência Nacional de Vigilância Sanitária (ANVISA), publicou o Regulamento Técnico de Boas Práticas para Serviços de Alimentação, de aplicabilidade em todo o território nacional, que estabelece a necessidade de inspeção e adequação das condições sanitárias, bem como o constante aprimoramento técnico de colaboradores deste meio (BRASIL,2004). 


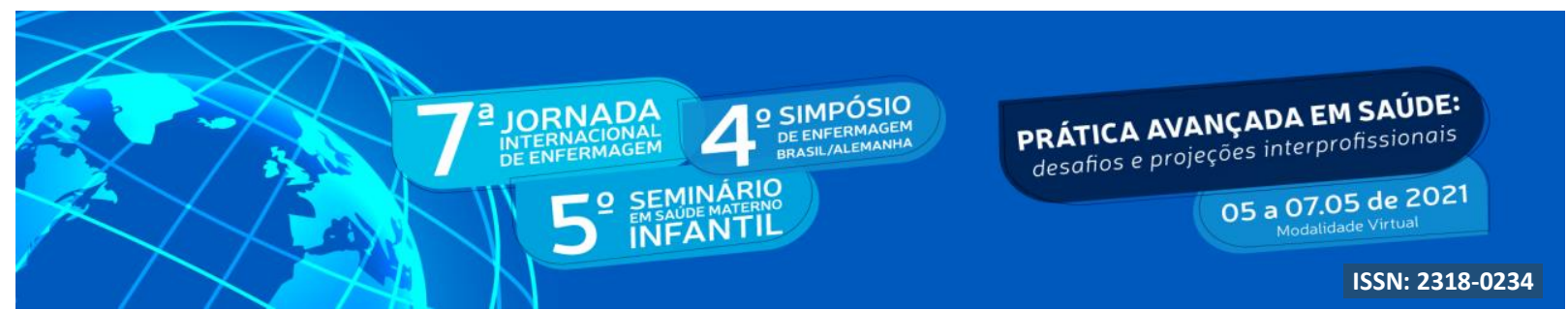

O Estado do Rio Grande do Sul possui uma legislação complementar a federal que institui que os manipuladores devem ser capacitados periodicamente, com frequência mínima anual, quanto aos aspectos relacionados a higiene pessoal, manipulação de alimentos e DTA. Essas capacitações devem ser comprovadas mediantes a comprovação (RIO GRANDE DO SUL, 2009). De acordo com Sangioni et al. (2019), a Portaria $n^{\circ}$. 78/2009 estabelece uma lista de verificação em BPM e definir normas a serem cumpridas pelo responsável pela manipulação.

Devido ao período pandêmico da covid-19, as atividades extensionistas dos cursos de graduação sofreram adaptações para a modalidade remota. Neste contexto, é de suma importância relatar as experiências acadêmicas obtidas nestas ações educativas de forma online junto aos manipuladores de alimentos.

\section{OBJETIVO}

O presente trabalho teve como objetivo relatar a experiência de uma disciplina extensionista ao promover uma capacitação online de Boas Práticas para manipuladores de alimentos e pequenos produtores de Santa Maria e região.

\section{METODOLOGIA}

Este trabalho trata-se de um relato de experiência dos acadêmicos do curso de nutrição da Universidade Franciscana de Santa Maria, matriculados na disciplina extensionista de Qualidade e Segurança dos Alimentos. Os estudantes desenvolveram uma capacitação em Boas Práticas de Manipulação (BPM), baseada nos conteúdos trabalhados em aula, na Portaria n. ${ }^{\circ}$ 78/2009 e na RDC n. ${ }^{\circ}$ 216/2004 (BRASIL, 2004; RIO GRANDE DO SUL, 2009), no mês de janeiro de 2021.

A organização e elaboração do cronograma e material didático, foi integrada ao Mestrado em Ciências da Saúde e da Vida da instituição de ensino. Foram criadas pequenas equipes para a organização das atividades de elaboração da capacitação, onde cada grupo ficou responsável 


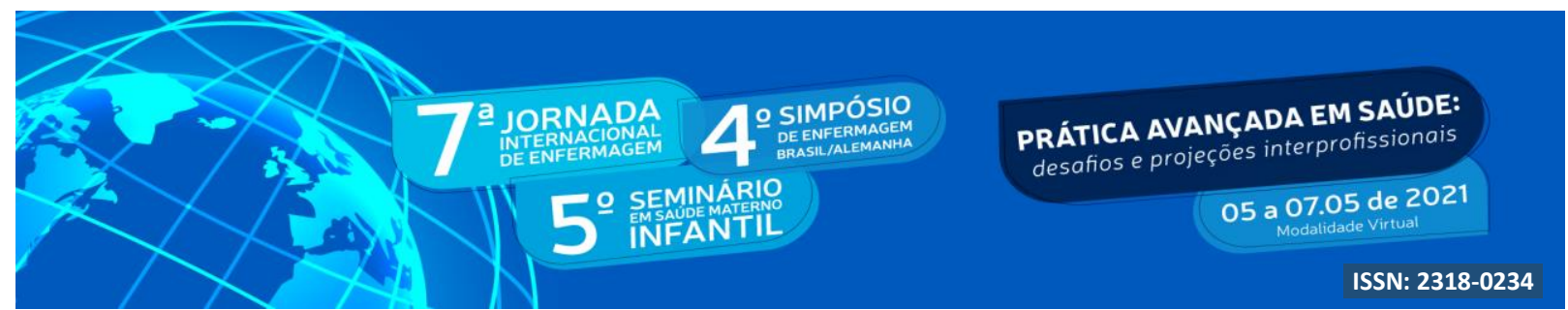

pela elaboração do flyer de divulgação, confecção do atestado de participação, definição das pautas a serem apresentadas e ficha de inscrição.

A divulgação aconteceu nas redes sociais dos organizadores, em matéria jornalística no Diário de Santa Maria e no site da instituição. O público-alvo foram os manipuladores de alimentos e pequenos produtores da região de Santa Maria.

As inscrições dos participantes foram feitas através de um link de formulário no Google Forms ${ }^{\circledR}$, que buscou coletar dados básicos como idade, gênero e qual o setor de manipulação operante. A capacitação ocorreu de forma online, na plataforma Google Meet®, com carga horária de 4 horas de duração, além de contar com atestado de participação e materiais didáticos elaborados pelos acadêmicos e posteriormente disponibilizados aos participantes do evento via $e$-mail. Ao final da capacitação também foi feito uma pesquisa de satisfação com os participantes pelo e-mail em um link do Google Forms ${ }^{\circledR}$, por meio da escala de Likert.

\section{RESULTADOS E DISCUSSÃO}

Na presente capacitação, 19 alunos da disciplina extensionista de Qualidade e Segurança dos Alimentos, foram divididos em grupos, desta maneira o conteúdo era abordado por um menor número de alunos. Os assuntos ministrados estão descritos conforme o quadro 1, cada tema contou com o apoio e auxílio da professora titular e de uma mestranda.

Quadro 1: Conteúdo programático da Capacitação em Boas Práticas na manipulação de alimentos, Santa Maria, 2021.

\begin{tabular}{|c|ll|}
\hline Grupos & \multicolumn{3}{|c|}{ Temas } \\
\hline Grupo 1 & Higiene Pessoal: Uniforme, Cabelo, unha, mãos e hábitos. \\
\hline Grupo 2 & $\begin{array}{l}\text { Higiene Ambiente: Etapas, produtos, diluições, utensílios adequados, } \\
\text { periodicidade, registros, panos e esponjas. }\end{array}$ \\
\hline Grupo 3 & Higiene dos alimentos: etapas, produtos e passo a passo. \\
\hline Grupo 4 & Recebimento e Armazenamento: Regras, cuidados, temperaturas e etiquetamento. \\
\hline Grupo 5 & Documentação e Legislação. \\
\hline
\end{tabular}




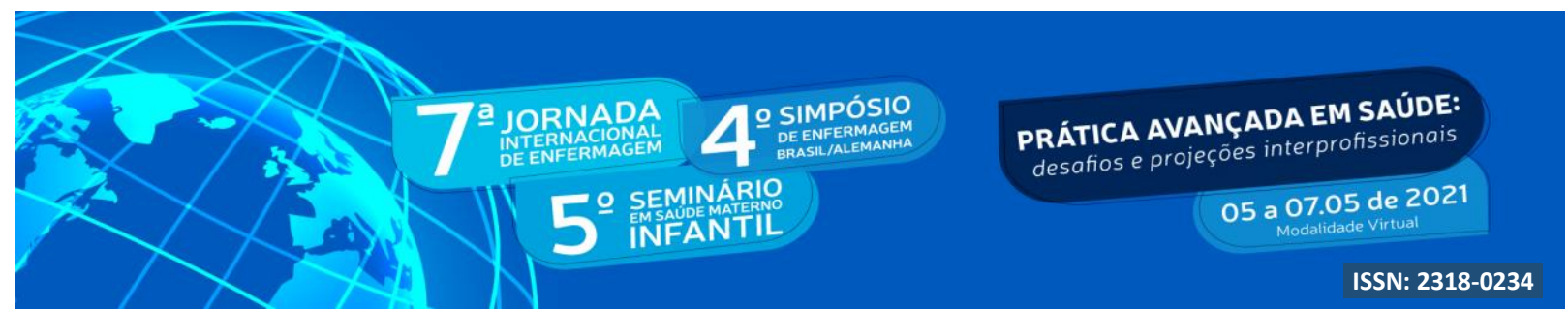

O evento teve divulgação nos canais de comunicação da instituição por um flyer criado pelos alunos, redes sociais (figura 1a) e matéria no Diário de Santa Maria (figura 1b). Entre nutricionistas, estudantes, manipuladores de alimentos, donos e frequentadores de restaurantes e pequenos produtores de Santa Maria, obteve-se 98 inscrições para o evento. Pôde-se constatar uma alta aceitabilidade do evento na forma remota quando comparado a mesma atividade extensionista realizada no ano de 2019 de forma presencial, onde obteve-se um número menor de inscritos e maior dificuldade de adesão.

Figura 1: Materiais utilizados para a divulgação Capacitação em Boas Práticas na manipulação de alimentos, Santa Maria, 2021.
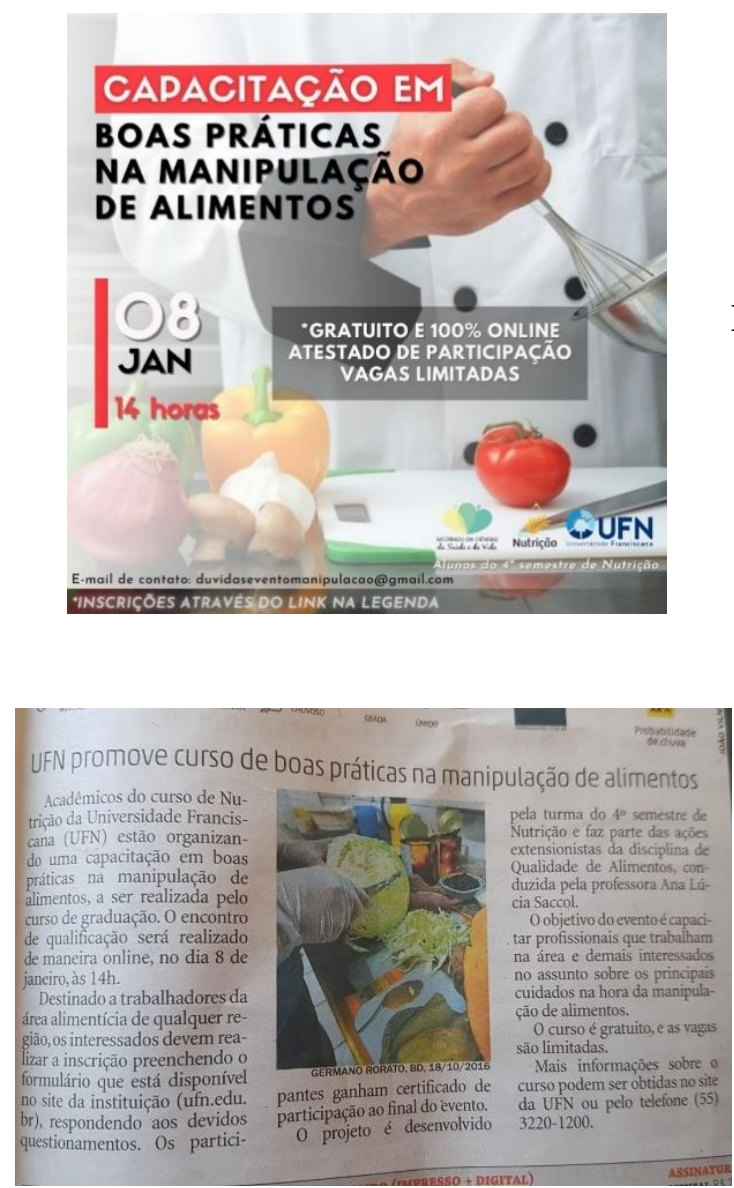

Figura 1a: Material elaborado pelos acadêmicos do curso de Nutrição -UFN

Figura 1b: Divulgação do Jornal Diário de Santa Maria 


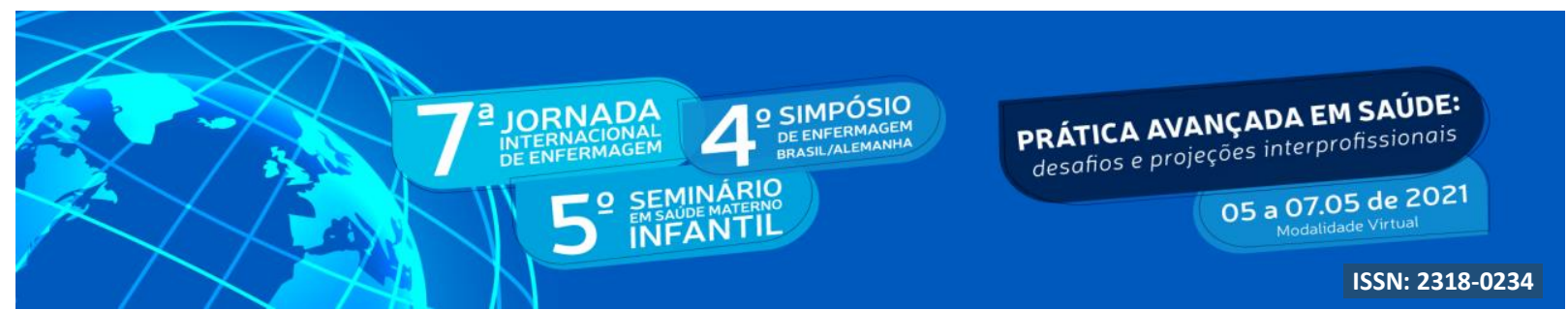

No início da atividade de capacitação, alguns alunos e participantes tiveram problemas de instabilidade de conexão e um dos ministrantes não conseguiu retornar às suas atividades. Apesar de alguns problemas técnicos, a atividade teve seguimento e pôde ser concluída com êxito.

Durante o andamento das apresentações, os participantes tinham liberdade para fazer perguntas e tirar suas dúvidas sobre as temáticas envolvidas. Foi elaborada uma pesquisa de satisfação (Figuras 2 e 3), a qual mostrou que o evento teve um excelente índice de aprovação e que os conteúdos abordados foram pertinentes em relação à adoção e execução das BPM.

Figura 2: Questão número um da pesquisa de satisfação dos participantes da Capacitação em Boas Práticas na manipulação de alimentos, sobre a programação, Santa Maria, 2021.

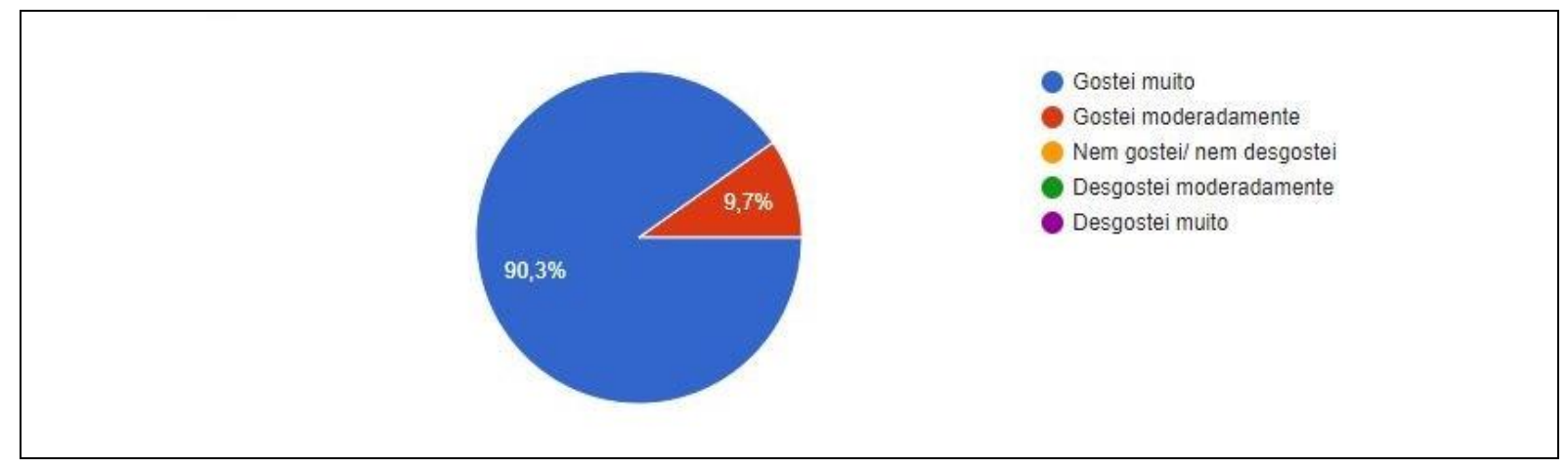

Figura 3: Questão número dois da pesquisa de satisfação dos participantes da Capacitação em Boas Práticas na manipulação de alimentos, quanto ao nível geral de satisfação, Santa Maria, 2021.

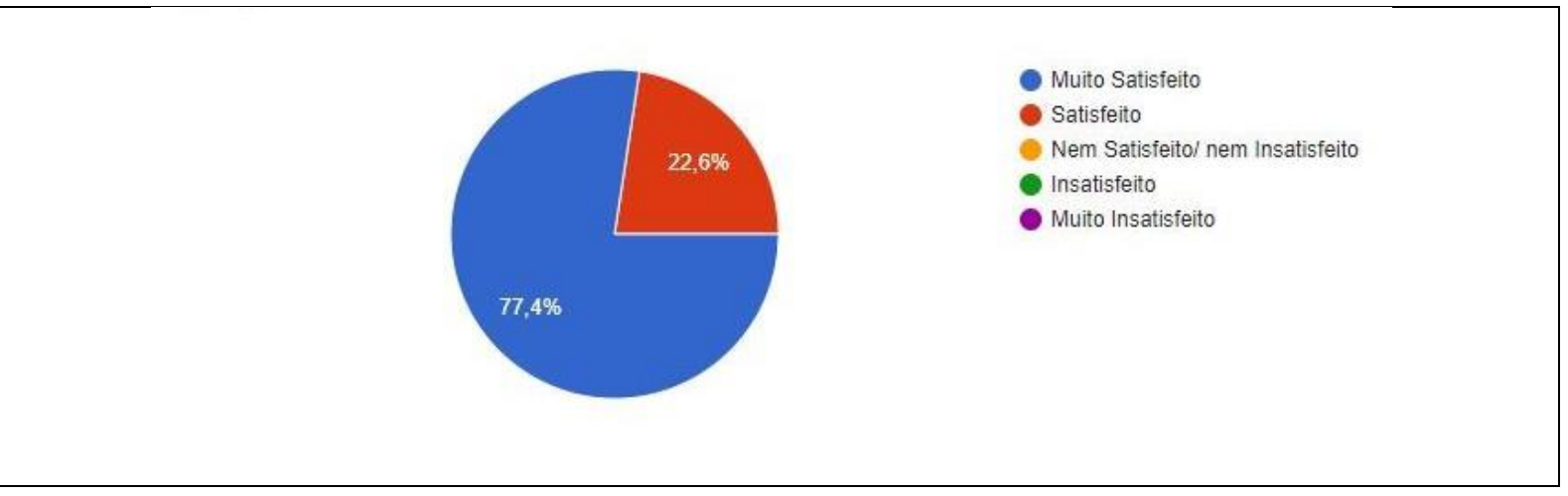




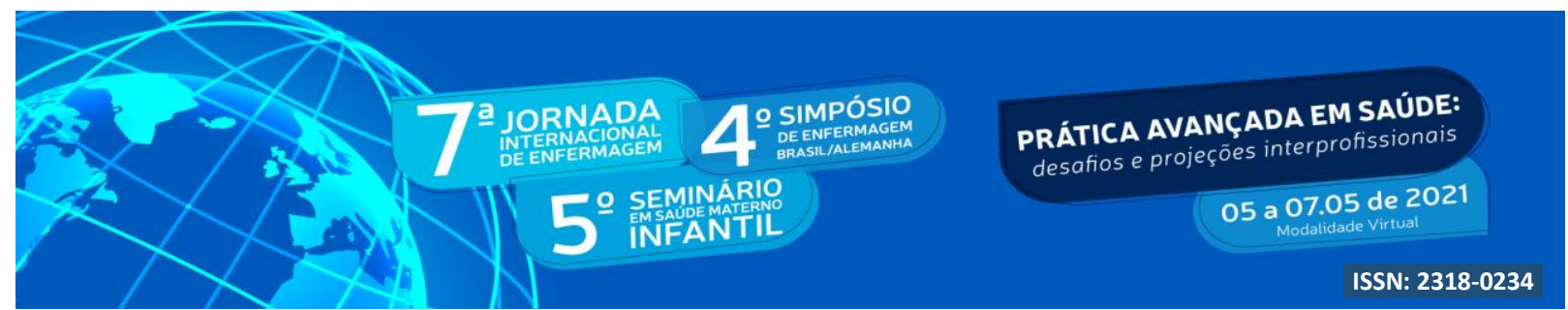

Desta maneira, é importante ressaltar que as qualificações se fazem necessárias, não somente pela exigência das legislações, mas também pela sua importância na formação destes profissionais. Por isso, foram disponibilizados atestados de conclusão (Figura 4) para todos os inscritos. Também é necessário relatar que para a maioria dos alunos, esta foi a primeira experiência de falar em público para pessoas externas à universidade. Com certeza um grande desafio proposto pela professora, mas que se faz necessário para uma boa formação e desenvoltura profissional.

Figura 4: Modelo de atestado entregue aos participantes, Capacitação em Boas práticas na Manipulação de Alimentos, Santa Maria, 2021.
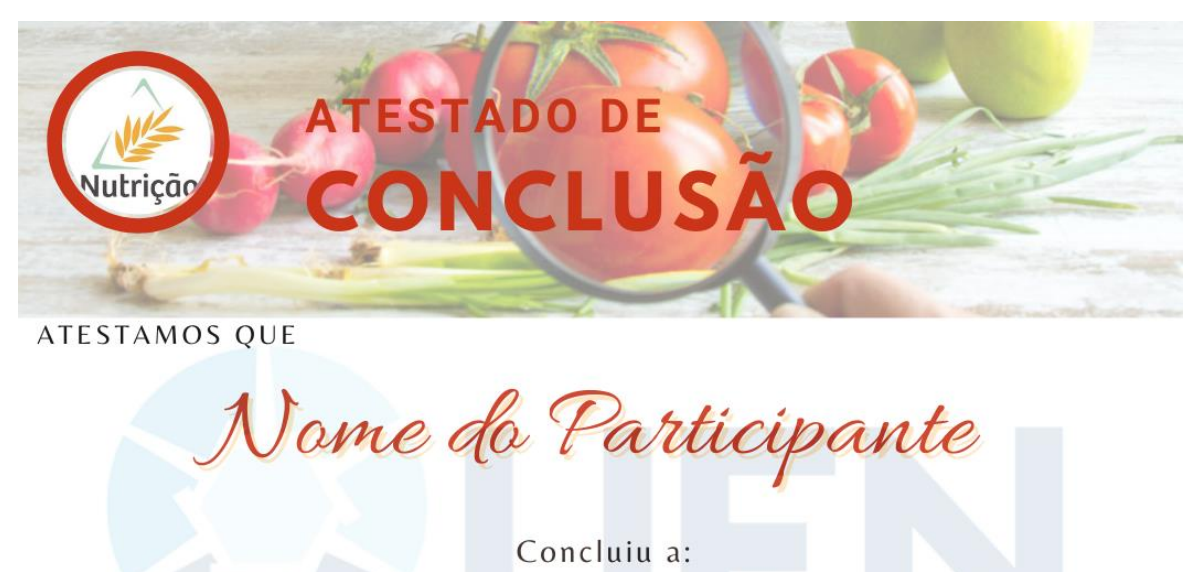

Capacitação em Boas Práticas na Manipulação de Alimentos, ministrada pelos alunos do $4^{\circ}$ semestre do Curso de Nutrição da Universidade Franciscana, no dia 08/01/2021, com carga horária de 4 horas.

Santa Maria - 08 de janeiro de 2021.

\section{CONCLUSÃO}

O estudo mostrou que apesar de imprevistos de conexão de alguns alunos, a capacitação ocorreu de forma tranquila e sem prejudicar a qualidade das abordagens, pois todos os alunos envolvidos estavam a par das temáticas de BPM. Além disso, a pesquisa de satisfação confirmou a expectativa dos organizadores, pois demonstrou excelente aceitabilidade pelos inscritos. 


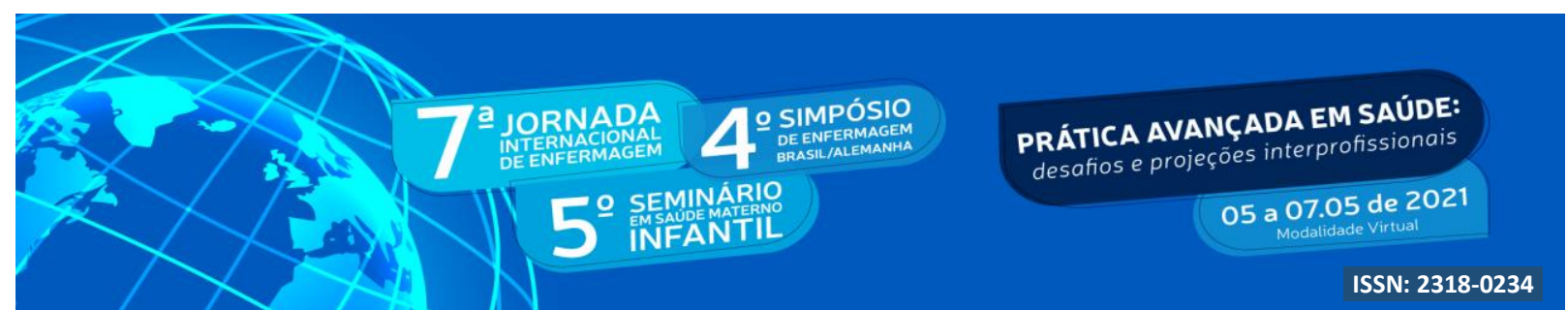

\section{REFERÊNCIAS}

ASSOCIAÇÃO BRASILEIRA DAS EMRESAS DE ALIMENTAÇÃO COLETIVA. Disponível em: \&lt;https://www.aberc.com.br/mercadoreal.asp?IDMenu=21\&gt; Acesso em: 29 de março. de 2021.

BEZERRA, I. N. et al. Eating away from home and excess weight: an analysis of explanatory mechanisms. Rev Bras Promoç Saúde, v. 29, n. 3, p. 455-461, 2016.

BRASIL. Ministério da Saúde. Doenças transmitidas por alimentos. Brasília, 2020.

Disponível em: https://www.gov.br/saude/pt-br/assuntos/saude-de-a-a-z-1/d/doencas$\underline{\text { transmitidas-por-alimentos; }}$

LIKERT, R., Roslow, S. \& Murphy, G. (1993). A simple and reliable method of scoring the Thurstone attitude scales. Personnel Psychology, vol. 46, p. 689-690. (Original publicado em 1934);

MINISTÉRIO DA SAÚDE. Resolução da Diretoria Colegiada n²16. Brasil, 2004. Disponível em:

https://bvsms.saude.gov.br/bvs/saudelegis/anvisa/2004/res0216_15_09_2004.html;

SANGIONI, LA, Cadore GC, Botton AS, Vogel FSF, Silva ERA, Smaniotto H, et al. Impactos do curso de boas práticas de manipulação de alimentos em estabelecimentos de serviços de alimentação de Santa Maria, Rio Grande do Sul.Vet. e Zootec. 2019:001-008

SECRETARIA DA SAÚDE. Portaria No78. Rio Grande do sul, 2009. Disponível em: http://www.tramandai.rs.gov.br/download/portaria_estadual_078_2009.pdf; 


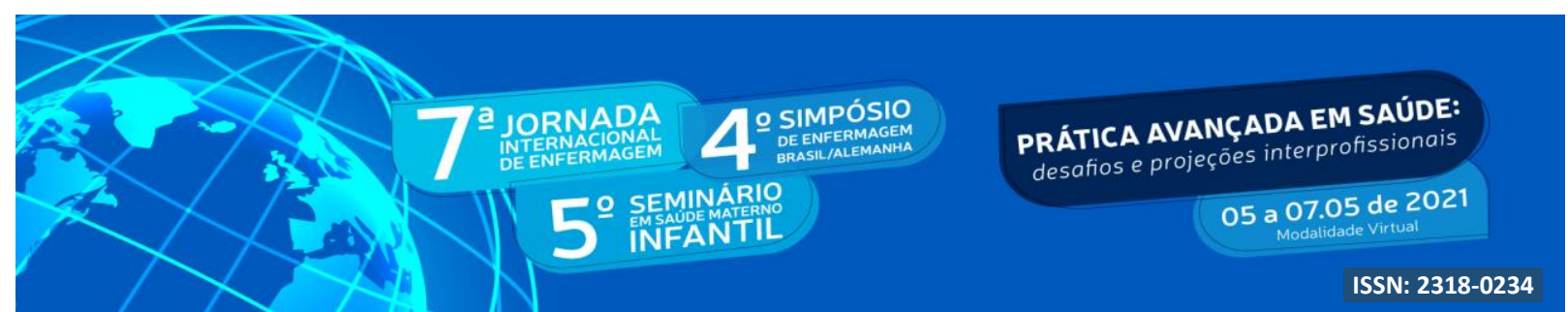

SOARES, S, Lilian. Conhecimento, atitudes e práticas de manipuladores de alimentos em segurança de alimentos: uma revisão sistemática. Higiene Alimentar. Vol. 30, n²56/257. 2016. Disponível em: https://docs.bvsalud.org/biblioref/2016/08/1473/separata-71-76.pdf.

TANIWAKI F, Baldini ED, Pereira JG, Silva RI, Frontana MSG, Jorge COA, Modolo JR. Importância de um curso de boas práticas de manipulação para manipuladores de alimentos em estabelecimentos de alimentação -Artigo de revisão. Vet 